\title{
Structures and Energetics of Models for the Active Site of Acetyl- Coenzyme A Synthase: Role of Distal and Proximal Metals in Catalysis
}

Charles Edwin Webster, Marcetta Y. Darensbourg, Paul A. Lindahl, and Michael B. Hall* Contribution from the Department of Chemistry, Texas A\&M University, College

Station, TX 77843-3255

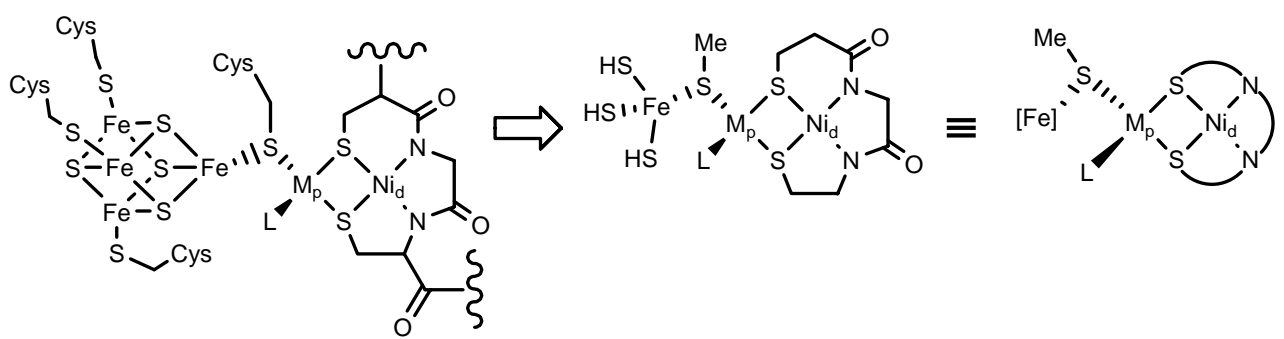

Scheme S1: Diagrammatic representation of the ACS active site and the model used in the current study

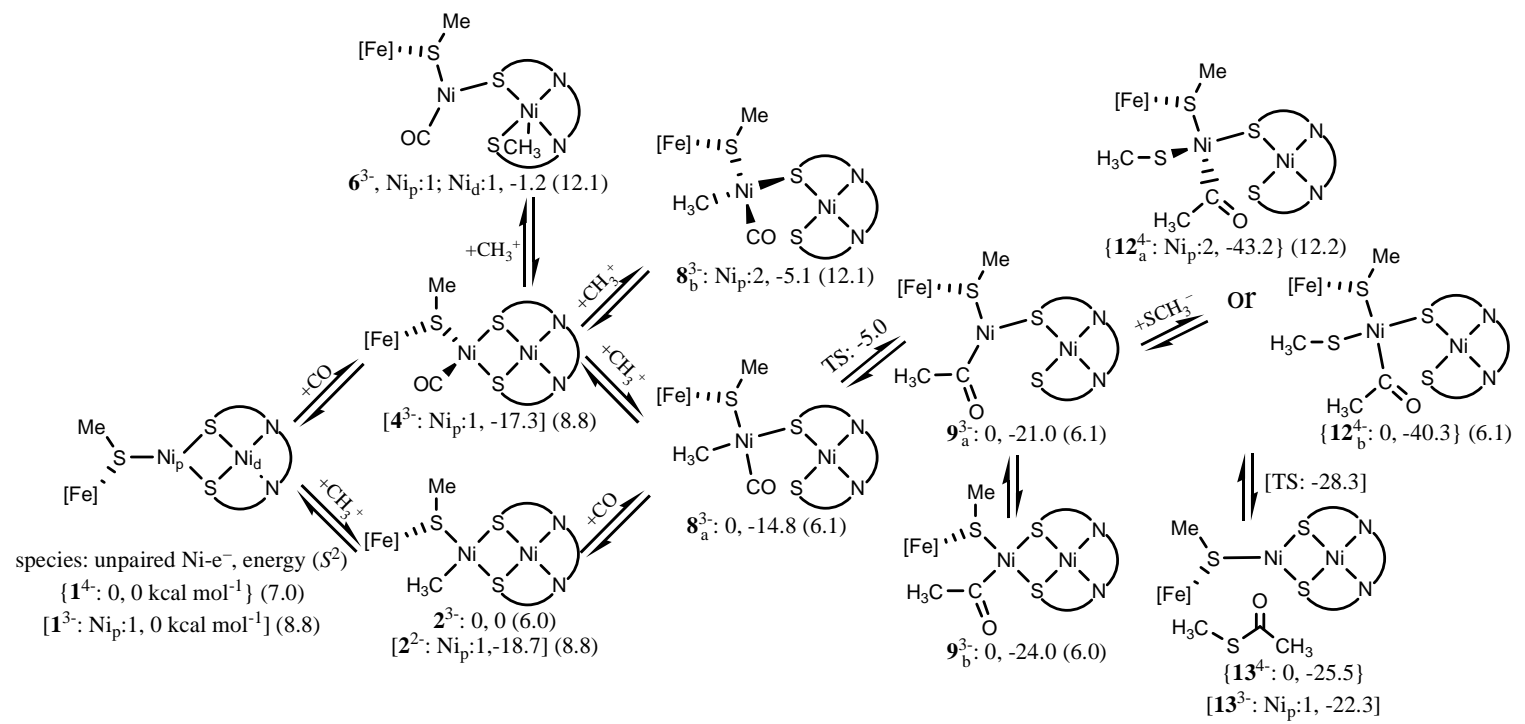

Scheme S2: Calculated energies $\left(\Delta \mathrm{E}_{0}\right)$ of optimized structures for intermediates and transition states. Labels refer to the species (as labeled in Table S1) with a superscript for the charge and a subscript for structural isomers. Unprojected $S^{2}$ values are in parenthesis after species designation (see discussion below); annihilation of higher spins did not significantly affect any of the $S^{2}$ values. All species contain high spin Fe". Three sets of relative energies are presented for species with different charges.

Table S1. Designations for and fates of the examined model species

\begin{tabular}{|c|c|c|c|c|c|c|c|}
\hline species $^{a}$ & ${ }^{2} \mathrm{Ni}_{\mathrm{p}}$ & $\mathrm{Ni}_{d}$ & ge & species $^{a}$ & $\mathrm{Ni}_{\mathrm{p}}$ & $\mathrm{Ni}_{d}$ & geometry \\
\hline $1^{4-}$ & - & - & stable & $7^{2-}$ & $\mathrm{Me}$ & $\mathrm{CO}$ & unstable \\
\hline $1^{3-}$ & - & - & stable & $8^{3-}$ & $(\mathrm{CO})(\mathrm{Me})$ & - & stable \\
\hline $2^{3-}$ & $\mathrm{Me}$ & - & stable & $8^{2-}$ & $(\mathrm{CO})(\mathrm{Me})$ & - & dissociates \\
\hline $2^{2-}$ & $\mathrm{Me}$ & - & stable & $9^{2-}$ & acetyl & - & stable \\
\hline $3^{3-}$ & - & $\mathrm{Me}$ & unstable & $9^{3-}$ & acetyl & - & stable \\
\hline $4^{4-}$ & $\mathrm{CO}$ & - & dissociates $^{b}$ & $10^{3-}$ & - & acetyl & unstable \\
\hline $4^{3-}$ & $\mathrm{CO}$ & - & stable & $11^{4-}$ & acetyl & S-Me & stable \\
\hline $5^{3-}$ & - & $\mathrm{CO}$ & unstable & $12^{4-}$ & (acetyl)(S-Me) & - & stable \\
\hline $6^{3-}$ & $\mathrm{CO}$ & $\mathrm{Me}$ & stable & $13^{4-}$ & - & - & stable \\
\hline $7^{3-}$ & $\mathrm{Me}$ & & unstable & $13^{3-}$ & - & - & stable \\
\hline
\end{tabular}

a. model number followed by species charge in superscript

b. dissociates by breaking the $\mathrm{Ni}_{\mathrm{p}}-\mathrm{S}_{\mathrm{Fe}}$ bond 
Gaussian $98^{1}$ implementation of B3LYP ${ }^{2,3}$ density functional theory; ${ }^{4}$ energies: pruned fine grids; gradients and Hessians: pruned course grids (unpruned for nickel, iron, and copper); geometry optimization SCF convergence: $10^{-6}$; geometries: fully optimized and analytical frequencies; basis sets: nickel, iron, and copper (341/341/41): Couty and Hall modification ${ }^{5}$ of Hay and Wadt LANL2DZ ${ }^{6}$ with effective core potentials (ECP); sulfur: standard Hay and Wadt LANL2DZ ${ }^{6 a}$ basis set with ECP supplemented with a $d$ polarization function; ${ }^{6 \mathrm{~b}}$ metal bound nitrogen and carbon and oxygen of CO: Dunning's cc-pVDZ ${ }^{7}$ (redundant functions removed and linearly transformed) ${ }^{8}$ all other carbon, oxygen, and hydrogen: D95V. ${ }^{9}$

Optimizations to minima and transition states were performed in redundant internal coordinates with the default Berny algorithm. In some instances, transition state optimizations were performed directly (i.e., OPT=TS, NOEIGEN), while, in other instances, quadratic synchronous transit methods were used (OPT=QST3, requiring three input geometries: two minima and a third geometry, an initial transition state structure).

All metal containing species were run unrestricted with high spin $\mathrm{Fe}^{\mathrm{II}}$ (i.e., 4 unpaired $\mathrm{e}^{-}$, as would be expected from the ligand field from four thiolate sulfurs). In certain instances, when indicated, other metals atoms have been calculated with unpaired electron density. Unprojected $S^{2}$ values are included in Scheme S2. Ideal $S^{2}$ values for 4 , 5 , and 6 unpaired electrons are $6,8.75$, and 12, respectively.

$$
S^{2}=\frac{n}{\mathbf{2}}\left(\frac{n}{\mathbf{2}}+\mathbf{1}\right) \text { where } n=\text { number of unpaired electrons }
$$

All relative energies are reported with corrections for the zero-point energy.

(1)Frisch, M. J.; Trucks, G. W.; Schlegel, H. B.; Scuseria, G. E..; Robb, M. A.; Cheeseman, J. R.; Zakrzewski, V. G.; Montgomery, J. A.; Stratmann, R. E.; Burant, J. C.; Dapprich, S.; Millam, J. M.; Daniels, A. D.; Kudin, K. N.; Strain, M. C.; Farkas, O.; Tomasi, J.; Barone, V.; Cossi, M.; Cammi, R.; Mennucci, B.; Pomelli, C.; Adamo, C.; Clifford, S.; Ochterski, J.; Petersson, G. A.; Ayala, P. Y.; Cui, Q.; Morokuma, K.; Malick, D. K.; Rabuck, A. D.; Raghavachari, K.;

Foresman, J. B.; Cioslowski, J.; Ortiz, J. V.; Stefanov, B. B.; Liu, G.; Liashenko, A.; Piskorz, P.; Komaromi, I.; Gomperts, R.; Martin, R. L.; Fox, D. J.; Keith, T.; Al-Laham, M. A.; Peng, C. Y.; Nanayakkara, A.; Gonzalez, C.; Challacombe, M.; Gill, P. M. W.; Johnson, B.; Chen, W.; Wong, M. W.; Andres, J. L.; Gonzalez, A. C.; Head-Gordon, M.; Replogle, E. S.; Pople, J. A. Gaussian 98, Revision A.11.3; Gaussian, Inc.: Pittsburgh, PA, 1998.

(2) Becke, A. D. J. Chem. Phys. 1993, 98, 5648-5652.

(3) Lee, C.; Yang, W.; Parr, R. G. Phys. Rev. B, 1988, 37, 785-789.

(4) Parr, R. G.; Yang, W. Density Functional Theory of Atoms and Molecules; Oxford University Press: New York, 1989.

(5) Couty, M.; Hall, M. B. J. Comp. Chem. 1996, 17, 1359-1370.

(6) (a) Hay, P. J.; Wadt, W. R. J. Chem. Phys. 1985, 82, 270-283; Wadt, W. R.; Hay, P. J. J. Chem. Phys. 1985, 82, 284-298; (b) Hollwarth, A.; Bohme, M.; Dapprich, S.; Ehlers, A. W.; Gobbi, A.; Jonas, V.; Kohler, K. F.; Stegmann, R.; Veldkamp, A.; Frenking, G. Chem. Phys. Lett. 1993, 208, 237-240; 1994, 224, 603.

(7) Dunning, T. H. J. Chem. Phys. 1989, 90, 1007-1023.

(8) Davidson, E. R. Chem. Phys. Lett. 1996, 260, 514-518.

(9) Dunning, T. H.; Hay, P. J. Modern Theoretical Chemistry, Ed. H. F. Schaefer, III, Plenum:

New York, 1976, pp 1-28. 